\title{
Brain tissue-derived autoimmune encephalitis cytokine TSLP primes neuroinflammation by activating JAK2-NLRP3 axis
}

\author{
Xueyuan $\mathrm{Yu}^{1}$, Jiajia $\mathrm{Lv}^{2}$, Jun $\mathrm{Wu}^{1}$, Yong $\mathrm{Chen}^{1}$, Fei Chen ${ }^{1}$, and Li Wang ${ }^{1}$ \\ ${ }^{1}$ Nanjing Brain Hospital \\ ${ }^{2}$ Shanghai Jiao Tong University Medical School Affiliated Ruijin Hospital
}

September 24, 2021

\begin{abstract}
Hyperactivation of NLRP3 inflammasome contributes to the neuroinflammation in autoimmune disorders, but the underlying regulating mechanism remains to be elucidated. We here demonstrate that mice lacking thymic stromal lymphopoietin receptor gene (Tslpr-/-) exhibit significant decreases in experimental autoimmune encephalitis (EAE) score, reduced CD4+ T cells infiltration, and restored expression of myelin basic protein (MBP) in the brain after induction of EAE by injection of myelin oligodendrocyte glycoprotein35-55 (MOG35-55) . TSLPR signals through Janus Kinase 2 (JAK2) to activate NLRP3. Tslpr-/mice of EAE show decreased phosphorylation of JAK2 and expression of NLRP3 in the brain. In wild type (WT) mice after induction of EAE, inhibition of JAK2 by ruxolitinib inflammatory and CD4+ cell infiltration, decreased expression of NLRP3, and restored BMP expression in the brain. Ruxolitinib also decreased levels of IL- $1 \beta$ and TSLP in brain of EAE mouse when compared to that without ruxolitinib treatment. Further results with NLRP3 inhibitor MCC950 in EAE mouse of WT verified the proinflammatory role of NLRP3 by showing decreased inflammatory cells and CD4+ T cells, restored MBP expression, and declined levels of IL- $1 \beta$ and TSLP in the brain. In patients with anti-N-methyl-D-aspartate receptor (NMDAR) encephalitis we found increased level of NLRP3 and IL-1 $\beta$ in CSF when compared to that in control subjects. These findings highlight TSLP as a prospective target for treating JAK2-NLRP3 axis-associated autoimmune inflammatory disorders.
\end{abstract}

\section{Introduction}

Experimental autoimmune encephalomyelitis (EAE), mediated by myelin-specific autoreactive T-helper cells, is a classical animal model of autoimmune encephalitis, such as anti-N-methyl-D-aspartate receptor (NMDAR) encephalitis, which manifested with typical demyelination and neurodegeneration-associated symptoms ${ }^{1}$. Although drugs targeting several immunological pathways have shown beneficial effects in patients with demyelinating disease, no cure is currently available ${ }^{2}$.

Nucleotide-binding domain, leucine-rich repeat containing protein family, pyrin domain containing 3 (NLRP3) and the IL-1 $\beta$ pathway have show to be crucial for the development of EAE by participating in the neuroinflammation ${ }^{3,4}$. Activation of NLRP3 comes from two ways, in which the first way is stimulation of pathogen recognition receptors that activate the nuclear factor $\chi \mathrm{B}(\mathrm{NF}-\varkappa \mathrm{B})$ pathways, and the second is activated by triggers such as lysosomal rupture, adenosine 5'-triphosphate (ATP), and reactive oxygen species $(\mathrm{ROS})^{5}$. Upon activation, NLRP3 inflammasome led to production of cleaved caspase-1 which cleaves proIL-1 $\beta /$ pro-IL-18 into mature IL-1 $\beta /$ IL-18 that induces cell pyroptosis-related massive cytokine release and systemic inflammation ${ }^{6}$. Potential mechanisms manipulated by NLRP3 to cause neuroinflammation include mediation of Th1 and Th17 responses ${ }^{7}$ and induction of chemotactic immune cell migration to the CNS ${ }^{8}$. NLRP3-depedent IL-1 $\beta$ maturation was reported to be detected in the lesions and cerebrospinal fluid (CSF) of MS patients ${ }^{9}$, and its presence strictly correlates with cortical lesion load ${ }^{10}$. Conversely, mice lacking IL-1 or with damaged IL-1 signaling showed compromised development and severity of EAE ${ }^{11}$. Only a few IL-1 targeted medicine are available for autoimmune disorders treatment ${ }^{12,13}$. Among these, JAK-STAT 
signaling is critical for $\mathrm{T}$ help cell polarization and autoimmune neuroinflammation ${ }^{14,}{ }^{15}$. In fact, several JAK inhibitors have been used over the past decades targeting a specific or a wide range of JAKs, providing promising alternatives to traditional biological disease modifying antirheumatic drugs in several autoimmune diseases ${ }^{16,17}$. Moreover, JAK mediate IL-4 receptor signals to prime Th2 response-dominant symptoms, like itch in atopic dermatitis ${ }^{18}$, in which cytokine IL-4 production is closely regulated by function of thymic stromal lymphopoietin ${ }^{19}$. Despite the high incidence and role of TSLP and JAK-STAT signaling, it is unclear whether TSLP functions via NLRP3 to induce autoimmune inflammation. If so, whether JAK is involved in this process is known.

Thymic stromal lymphopoietin (TSLP) is an interleukin (IL)-7 related cytokine acting on lineages, including macrophages, dendritic cells (DCs), and T cells ${ }^{20}$. By promoting expression of major histocompatibility complex (MHC)-II and co-stimulatory molecules such as CD40, CD80, and CD86, and the production of chemokines, TSLP strongly enhances DCs maturation and function ${ }^{21}$. MS and EAE have been associated with single nucleotide polymorphisms (SNPs) in IL-7Ragene locus ${ }^{22}$. Upon ligation by TSLP, TSLP receptor (TSLPR) initiates intracellular JAK/STAT signaling to induce production of IL-2, TNF and IL-6 to potentiate inflammatory responses. Targeting these cytokines has been shown to be effective in alleviating EAE or other autoimmune disease ${ }^{23,24,25,26}$. Accordingly, direct blocking JAK/STAT signaling pathways with tofacitinib inhibited NLRP3 inflammasome and IL-1 $\beta$ production in neutrophils ${ }^{27}$. However, whether TSLPR signaling is capable of controlling initiates inflammation and in EAE remains unclear.

In this study we show that $T s l p r^{-/-}$mice presented alleviated severity in myelin oligodendrocyte glycoprotein peptide $\left(\mathrm{MOG}_{35-55}\right)$-induced experimental autoimmune encephalitis (EAE), resulting from decreased phosphorylation of JAK2 and expression of NLRP3. Inhibition of JAK by ruxolitinib reduced NLRP3 expression in brain of EAE mouse. In addition, using JAK inhibitor ruxolitinib or NLRP3 inhibitor MCC950 all reduced inflammatory cells and CD4 ${ }^{+}$cells infiltration, NLRP 3 and myelin expression, and IL- $1 \beta$ and TSLP in brain tissue of EAE mouse. Furthermore, patients with anti-N-methyl-D-aspartate-receptor (anti-NMDAR) encephalitis showed a significant increase inNLRP3 and IL-13in CSF when compared with that in healthy control. These findings reveal that TSLP plays an essential role in positive regulation of JAK2-NLRP3 axis-driven neuroinflammation in autoimmune disorders.

\section{Materials and methods}

\section{Patients and Controls}

We recruited 5 patients diagnosed of anti-NMDAR encephalitis (all patients with anti-NMDAR antibody positive) and 7 controls from Nanjing Brain Hospital. The anti-NMDAR encephalitis diagnosed criteria of 2016 were chosen as the inclusive criteria for the group of patients with anti-NMDAR encephalitis ${ }^{28}$. These control subjects consisted of patients with 6 patients with hydrocephalus and 1 patient with acute head trauma and all of them have no autoimmune encephalitis. The baseline characteristics of patients $(\mathrm{n}=5)$ and controls $(\mathrm{n}=7)$ were shown in Table 1 .

This study was approved by the Ethics Committee of Nanjing Brain Hospital, Nanjing Medical University.

\section{EAE induction and scoring}

$\mathrm{Tslpr}^{-/-}$mice and WT mice were bred under specific pathogen-free (SPF) conditions. Tslpr ${ }^{-/}$mice were purchased from Shanghai Biomodel Organism Science \& Technology Development Co.,Ltd. FemaleTslpr /- $^{-}$ and WT mice (10-12 weeks old) were immunized subcutaneously (s.c.) with $\mathrm{MOG}_{35-55}$ (Beyotime, China) with $4 \mathrm{mg} / \mathrm{ml}$ heat-inactivated Mycobacterium tuberculosis H37Ra (BD) at day 1 to induce EAE as described before ${ }^{29} .200 \mathrm{ng} /$ mouse pertussis toxin (List Biological Laboratories Inc.) was injected i.p. at day 0 and day 2. Mice were sacrificed at day 15 and brain tissue was collected for western blot or immunohistochemistry. Symptoms of EAE paralysis in mice was scored as follows: 0, no disease; 1, tail weakness; 2, paraparesis; 3, paraplegia; 4, paraplegia with forelimb weakness; 5 , moribund or dead animals. To evaluate the contribution of NLRP3 inflammasome or JAK to CNS inflammation, i.p. injection of $50 \mathrm{mg} / \mathrm{kg}$ MCC950 (dissolved in DMSO) ${ }^{30}$ or oral administration of ruxolitinib $(90 \mathrm{mg} / \mathrm{kg} / \mathrm{d}){ }^{31}$ after $\mathrm{MOG}_{35-55}$ immunization 
were performed.

\section{CNS inflammation}

For determination of inflammation infiltrates, brains were harvested, fixed in $4 \%$ formalin stored at room temperature. Tissue histology, including HE, NLRP3 staining, CD4 staining, and Luxol Fast Blue (LFB) staining (Servicebio, China) of brain tissue sections was performed to determine the infiltrates of inflammatory cells, $\mathrm{CD} 4^{+} \mathrm{T}$ cells and myelin sheath expression.

\section{Western blot}

Determination of NLRP3, JAK2 phosphorylation (CST, USA), basic meylin protein (MBP), $\beta$-actin, and GAPDH (Servicebio, China) in brain tissue of mice by western blot was performed as we previously described $^{32}$.

\section{ELISA}

Determination of NLRP3 inflammasome (ELISAGenie, UK) and IL-1 $\beta$ (DAKEWE, China) in human CSF, and IL-1 $\beta$ and TSLP (Biolegend, USA) in mouse brain tissue homogenate was performed according to the manufacture's instruction.

\section{Statistical analysis}

Statistical significance was determined using unpaired two-tailed Student's $t$-test to perform statistical analysis of all data (GraphPad Prism version 5.0; GraphPad Software). $p<0.05$ was considered statistically significant. Data are expressed as the mean \pm standard error of the mean (SEM).

\section{Results}

$\mathrm{Tslpr}^{-/-}$mice show decreased neuroinflammation in EAE model

$\mathrm{MOG}_{35-55}$ was used to induce EAE mice model (Fig. 1A). To investigate the role of TSLPR in CNS autoimmunity, the clinical score of EAE in Tslpr ${ }^{-/}$and $T s l p r^{+/+}$mice was determined. After EAE induction, $T s l p r+/+$ mice developed into typical monophasic EAE symptoms manifested with ascending paralysis 1012 days after $\mathrm{MOG}_{35-55}$ imunization (Fig. 1B). By contrast, Tslpr ${ }^{-/}$mice show delayed onset of paralysis at 11-13 days and alleviated symptoms when compared to that in $T s l p r+/+$ mice. Even though, EAE score of both group peaked at day 15 (Fig. 1B). In addition, immunohistochemistry analysis of brain tissues from Tslpr ${ }^{+/+}$and $T s l p r^{-/-}$mice displayed that at day 15 after EAE induction revealed significant reductions in the number of $\mathrm{CD}^{+}$lymphocytes present as inflammatory infiltrates of the brain tissue of $\mathrm{Tslpr}^{-/-}$mice when compared to that in Tslpr ${ }^{+/+}$mice (Fig. 1C). Importantly, results from western blot revealed that mice MOG35-55 injection led to reduced expression of myelin basic protein (MBP) in Tslpr $+/+$ mice when compared to that without $\mathrm{MOG}_{35-55}$ injection. However, Tslpr ${ }^{-/-}$mice of EAE showed restored expression of MBP when compared to that in Tslpr $+/+$ mice (Fig. 1D).

\section{TSLPR signals via JAK2 and NLRP3}

It has been recently demonstrated that the development of EAE requires NLRP3 ${ }^{8}$. As we found above that TSLPR deficiency results in reduced $\mathrm{CD} 4^{+} \mathrm{T}$ lymphocytes infiltration in EAE development, we determined to examine whether TSLPR signaling requires NLRP3 inflammasome activation in EAE. Results showed that $\mathrm{MOG}_{35-55}$ treatment resulted in increased phosphorylation of JAK2 and expression of NLRP3 when compared to that in control mice (Fig 2A). Immunohistochemistry analysis of brain tissues from Tslpr ${ }^{+/+}$and Tslpr $^{-/-}$ mice showed that $\mathrm{MOG}_{35-55}$ injection led to remarkable increase in $\mathrm{NLRP}^{+}$cells in the brain in Tslpr ${ }^{+/+}$ mice when compared to that without $\mathrm{MOG}_{35-55}$ injection while a significant reduction in $\mathrm{NLRP}^{+}$cells was observed in brain tissue of $\mathrm{Tslpr}^{-/-}$mice when compared to that in $\mathrm{Tslpr}^{+/+}$mice after EAE induction (Fig 2B).

\section{NLRP3 is involved in JAK2-associated neuroinflammation}


TSLPR ligation activates JAK, specifically JAK1 and JAK2 but not JAK3, in primary T cells ${ }^{32,}{ }^{33}$. To further explore the role of JAK2 that mediates neuroinflammation, we applied JAK inhibitor, which is the selective and orally bioavailable JAK1/2 inhibitor widely used in myelofibrosis, to block JAK signaling. Treatment of ruxolitinib by oral administration in EAE mice resulted in significant reduction in inflammatory cells infiltration by HE staining (Fig 3A), CD4 ${ }^{+} \mathrm{T}$ cells infiltration (Fig 3B), and ultimate restoration of myelin sheath expression by Luxol Fast Blue staining (LFB) (Fig 3C) and MBP expression by western blot (Fig 3D). JAK inhibition by ruxolitinib also decreased expression of NLRP3 when compared to that without JAK inhibition in brain of EAE mice (Fig 3D). ELISA experiments show that JAK inhibition reduced IL-1 $\beta$ level in the brain which confirms NLRP3 hyporeactivity (Fig 3E). ELISA experiments also show reduced TSLP in the brain after JAK inhibition in EAE mice when compared to that without JAK inhibition (Fig 3F). Together, our data demonstrate that JAK2 mediates neuroinflammation via NLRP3.

\section{NLRP3 inhibition alleviates neuroinflammation}

To evaluate the role of NLRP3 in the neuroinflammation of EAE, MCC950, a potent, selective, small-molecule inhibitor of NLRP3, was used to block canonical and noncanonical NLRP3 activation ${ }^{30}$. Treatment of MCC950 by i.p administration in EAE mice resulted in significant reduction in inflammatory cells infiltration by HE staining (Fig 4A), CD4 ${ }^{+}$cells infiltration (Fig 4B), and restoration of myelin sheath expression by LFB staining (Fig 4C) and MBP by western blot (Fig 4D). NLRP3 inhibition also reduced IL-1 $\beta$ level (Fig $4 \mathrm{E}$ ) and TSLP level (Fig $4 \mathrm{~F}$ ) in the brain of EAE mice when compared to that without NLRP3 inhibition.

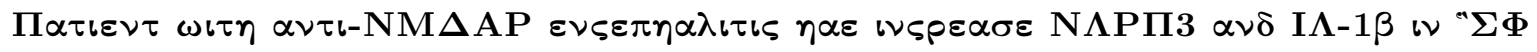

To verify NLRP3 activation in the brain, NLRP3 inflammasome and IL-1 $\beta$ were detected in patients with anti-NMDAR encephalitis and in control subjects. Clinical features of control subjects and patients with anti-NMDAR encephalitis are present in Table 1. Results showed that levels of NLRP3 inflammasome (Fig $5 \mathrm{~A}$ ) and IL-1 $\beta$ (Fig 5B) were remarkably increased in patients with anti-NMDAR encephalitis when compared to that in controls.

\section{Discussion}

Activation of NLRP3 is a tightly regulated process and a key step in autoimmunity of CNS. In this study, we provide three advances that broaden our understanding of NLRP3-associated neuroinflammation. First, by using $\mathrm{Tslpr}^{-/-}$mice, we demonstrated that TSLP signaling regulates neuroinflammation and paralysis of mouse. Second, we show that TSLPR signals via JAK2 to activate NLRP3-associated neuroinflammation in EAE settings. Third, we show that patients with anti-NMDAR encephalitis have increased levels of NLRP3 and IL-1 $\beta$ in CSF. Taken together, the current study identifies novel functions of TSLP-dominant JAK pathways upstream of NLRP3 represent promising targets for the treatment of autoimmune disorders.

Generally, Th17 response in autoimmune disorders has been believed to cause neurons death or inflammatory response ${ }^{34,35}$. Besides, previous studies also have shown that type 3 innate lymphoid cell (ILC3) produce IL-17 in autoimmune disorder such as ankylosing spondylitis ${ }^{36}$ and that ILC3 can maintains neuroinflammation by supporting $\mathrm{T}$ cell survival ${ }^{37}$. Thus, both neuroinflammation and demyelination were largely believed to be mediated by Th17- and ILC3- related responses. We and other have recently shown that TSLP primes dendritic cells (DCs) maturation ${ }^{21,38}$ and that DCs determines status of activation and survival of ILC3 ${ }^{39}$. Based on our new data demonstrating that brain tissue TSLPR signaling critically mediates neuroinflammation, we speculate that innate immune cells, such as ILC3s, play important roles in promoting autoimmune inflammation-associated demyelination, in addition to Th17 cells. Identification of the precise role of TSLP in Th17 cells- and ILC3-mediated neuroinflammation and demyelination requires further investigation.

Despite previous observation that TSLP is reported to be critical for regulatory T cells formation in autoimmune disease, we found that, in contrast to decreased IL-7R expression on conventional T cells and reduced Treg function of MS in previous study ${ }^{40}$, deletion of Tslpr efficiently alleviated neuroinflammation displayed by decreased $\mathrm{CD} 4^{+}$cells infiltration and restored myelin expression, which coincides with one previous study 
showing ameliorated EAE symptoms accompanied by reduced inflammatory infiltrates in the brain of Tslp -/- mice $^{41}$. In fact, the epithelial cell-derived cytokine TSLP, GM-CSF, and IL-25 have been shown to be master initiators of type 3 inflammation via their effects on a variety of cells including Th17, ILC3, and mast cells ${ }^{42,43,44}$. These cytokines are believed to rapidly bind to membrane receptor in order to generate innate immune response and therefore prime adaptive immune cells. Strikingly, two recent studies have demonstrated that TSLP directly activates neurons ${ }^{45,46}$. Additionally, our current data highly regulated TSLP in the brain in the settings of EAE coincides with previous study ${ }^{47}$ and go beyond by demonstrating that TSLP receptor signals by phosphorylation of JAK2 to activate NLRP3-mediated inflammation in response to $\mathrm{MOG}_{35-55}$ administration. Thus, we speculate that TSLP cytokine may act as master regulator of neuroinflammation in immune cells of brain.

In immune cells, cytokine signaling by the JAK-STAT pathway causes transcriptional changes to promote cellular activation. However, although JAK inhibitor has been reported to be an alternative immunotherapy in patients with autoimmune disorders such as neuromyelitis optica ${ }^{48}$, our data indicated that neuroinflammation to MOG35-55 injection with additional JAK inhibition by ruxolitinib failed to induce typical neuroinflammation as observed in EAE mice brain. Also, these decreased neuroinflammation was accompanied by restored expression of myelin basic protein after treatment with ruxolitinib. Thus, we predict that alterations in classic JAK-mediated NLRP3 inflammasome activation is sufficient to explain how neuroinflammation and demyelination occur. One previous study has shown that JAK1 mediates sensory neuronal responsiveness which can be enhanced by cytokines such as IL-4 ${ }^{18}$. Our data coincides with this study and further demonstrates that JAK proteins have novel functions in neurons and regulates meylination/demyelination balance at least by NLRP3-mediated pathways. However, we note that such alteration of myelin basic protein expression does not exclude the role of JAK2 or other pathways in modulating transcription or other post-transcription of myelin basic protein within CNS. Future studies will be required to better understand how changes of JAK-STAT pathway impact myelin expression and neuroinflammation in autoimmune disorders.

Clinical application for ruxolitinib, an non-selective JAK1/2 inhibitor, have been reported to induce improvement of neurologic disability in neuromyelitis optica ${ }^{48}$. In fact, significant clinical efficacy of symptoms in autoimmune disorders has been observed in clinical trials employing other JAK inhibitor such as tofacitinib and barcitinib ${ }^{49,50}$. Previously, the changes in neuroinflammation observed with JAK inhibition have been attributed to the anti-inflammatory role of Th17 response ${ }^{35}$. Recently, study from others demonstrate that transient receptor potential (TRP) plays a critical role in EAE by mediating axonal and neuronal degeneration ${ }^{51}$ and that JAK-STAT pathway determines TRP expression ${ }^{52,53}$, which indicates involvement of TRP in JAK pathway-mediated neuroinflammation. Based on our and others' studies, we speculate that the improvement of neuroinflammation in EAE mice treated with ruxolitinib may be mediated, at least in part, by disruption of these signals in the CNS and that these therapies may alleviate neuroinflammation in autoimmune disorders. Strikingly, recent studies published experimental and clinical evidence for evobrutinib, the first Bruton's tyrosine kinase (BTK) inhibiting molecule being developed, also described reduced disease severity in clinical and mouse model of multiple sclerosis (MS) by impairment of encephalitogenic T cells ${ }^{54,55}$. Given our current study demonstrating a direct role of JAK in neuroinflammation, whether combinations of TSLP-, JAK-, and BTK-blockade can lead to synergistic therapeutic improvements of neuroinflammation in autoimmune disorders such as MS demands further investigation.

In conclusion, our data establish and highlight the capability of TSLPR-JAK signaling inhibition to control disease-driving neuroinflamamtion of NLRP3 in inflammatory CNS demyelination. This is demonstrated here for ruxolitinib, a non-selective JAK inhibitor clinically tested in several autoimmune diseases, and the elucidated immunological effects may similar apply to other JAK inhibitors in clinical development. Based on this, the mechanistic data provided here will be instrumental in facilitate how this molecule is integrated into the current autoimmune disorder treatment.

\section{References}

1. Titulaer, M.J. et al. Overlapping demyelinating syndromes and anti-N-methyl-D-aspartate receptor 
encephalitis. Annals of neurology 75 , 411-428 (2014).

2. Comi, G., Radaelli, M. \& Soelberg Sørensen, P. Evolving concepts in the treatment of relapsing multiple sclerosis. Lancet (London, England) 389 , 1347-1356 (2017).

3. Shi, H. et al. NLRP3 activation and mitosis are mutually exclusive events coordinated by NEK7, a new inflammasome component. Nature immunology 17 , 250-258 (2016).

4. Chivero, E.T. et al. HIV-1 Tat Primes and Activates Microglial NLRP3 Inflammasome-Mediated Neuroinflammation. The Journal of neuroscience : the official journal of the Society for Neuroscience37 , 3599-3609 (2017).

5. Rathinam, V.A. \& Fitzgerald, K.A. Inflammasome Complexes: Emerging Mechanisms and Effector Functions. Cell 165 , 792-800 (2016).

6. Miao, E.A., Rajan, J.V. \& Aderem, A. Caspase-1-induced pyroptotic cell death. Immunological reviews 243, 206-214 (2011).

7. Gris, D. et al. NLRP3 plays a critical role in the development of experimental autoimmune encephalomyelitis by mediating Th1 and Th17 responses. Journal of immunology (Baltimore, Md. : 1950)185, 974-981 (2010).

8. Inoue, M., Williams, K.L., Gunn, M.D. \& Shinohara, M.L. NLRP3 inflammasome induces chemotactic immune cell migration to the CNS in experimental autoimmune encephalomyelitis. Proceedings of the National Academy of Sciences of the United States of America109 , 10480-10485 (2012).

9. Halle, A. et al. The NALP3 inflammasome is involved in the innate immune response to amyloid-beta. Nature immunology 9 , 857-865 (2008).

10. Seppi, D. et al. Cerebrospinal fluid IL-1 $\beta$ correlates with cortical pathology load in multiple sclerosis at clinical onset.Journal of neuroimmunology 270 , 56-60 (2014).

11. Hauptmann, J. et al. Interleukin-1 promotes autoimmune neuroinflammation by suppressing endothelial heme oxygenase-1 at the blood-brain barrier. Acta neuropathologica 140 , 549-567 (2020).

12. Mellergård, J., Edström, M., Vrethem, M., Ernerudh, J. \& Dahle, C. Natalizumab treatment in multiple sclerosis: marked decline of chemokines and cytokines in cerebrospinal fluid. Multiple sclerosis (Houndmills, Basingstoke, England) 16 , 208-217 (2010).

13. Carpintero, R. \& Burger, D. IFN $\beta$ and glatiramer acetate trigger different signaling pathways to regulate the IL-1 system in multiple sclerosis. Communicative $\&$ integrative biology 4 , 112-114 (2011).

14. Garris, C.S. et al. Defective sphingosine 1-phosphate receptor 1 (S1P1) phosphorylation exacerbates TH17-mediated autoimmune neuroinflammation. Nature immunology 14, 1166-1172 (2013).

15. Wang, X. et al. OTUB1 inhibits CNS autoimmunity by preventing IFN- $\gamma$-induced hyperactivation of astrocytes. The EMBO journal38 (2019).

16. Damsky, W. et al. The emerging role of Janus kinase inhibitors in the treatment of autoimmune and inflammatory diseases. The Journal of allergy and clinical immunology 147, 814-826 (2021).

17. Baker, K.F. \& Isaacs, J.D. Novel therapies for immune-mediated inflammatory diseases: What can we learn from their use in rheumatoid arthritis, spondyloarthritis, systemic lupus erythematosus, psoriasis, Crohn's disease and ulcerative colitis? Annals of the rheumatic diseases 77 , 175-187 (2018).

18. Oetjen, L.K. et al. Sensory Neurons Co-opt Classical Immune Signaling Pathways to Mediate Chronic Itch. Cell 171, 217-228.e213 (2017).

19. Hussain, M. et al. Basophil-derived IL-4 promotes epicutaneous antigen sensitization concomitant with the development of food allergy. The Journal of allergy and clinical immunology141, 223-234.e225 (2018). 
20. Corren, J. \& Ziegler, S.F. TSLP: from allergy to cancer.Nature immunology 20 , 1603-1609 (2019).

21. Liang, Y. et al. Thymic stromal lymphopoietin epigenetically upregulates Fc receptor $\gamma$ subunit-related receptors on antigen-presenting cells and induces $\mathrm{T}(\mathrm{H}) 2 / \mathrm{T}(\mathrm{H}) 17$ polarization through dectin-2. The Journal of allergy and clinical immunology 144 , 1025-1035.e1027 (2019).

22. Gregory, S.G. et al. Interleukin 7 receptor alpha chain (IL7R) shows allelic and functional association with multiple sclerosis.Nature genetics 39 , 1083-1091 (2007).

23. Samoilova, E.B., Horton, J.L., Hilliard, B., Liu, T.S. \& Chen, Y. IL-6-deficient mice are resistant to experimental autoimmune encephalomyelitis: roles of IL-6 in the activation and differentiation of autoreactive T cells. Journal of immunology (Baltimore, Md. : 1950) 161, 6480-6486 (1998).

24. Duong, T.T., St Louis, J., Gilbert, J.J., Finkelman, F.D. \& Strejan, G.H. Effect of anti-interferon-gamma and anti-interleukin-2 monoclonal antibody treatment on the development of actively and passively induced experimental allergic encephalomyelitis in the SJL/J mouse. Journal of neuroimmunology 36 , 105-115 (1992).

25. Baker, D. et al. Control of established experimental allergic encephalomyelitis by inhibition of tumor necrosis factor (TNF) activity within the central nervous system using monoclonal antibodies and TNF receptor-immunoglobulin fusion proteins. European journal of immunology 24, 2040-2048 (1994).

26. Hartgring, S.A. et al. Critical proinflammatory role of thymic stromal lymphopoietin and its receptor in experimental autoimmune arthritis. Arthritis and rheumatism 63, 1878-1887 (2011).

27. Furuya, M.Y. et al. Tofacitinib inhibits granulocyte-macrophage colony-stimulating factor-induced NLRP3 inflammasome activation in human neutrophils. Arthritis research $\mathbb{E}$ therapy 20 , 196 (2018).

28. Graus, F. et al. A clinical approach to diagnosis of autoimmune encephalitis. The Lancet. Neurology 15 , 391-404 (2016).

29. Lévesque, S.A. et al. Myeloid cell transmigration across the CNS vasculature triggers IL-1 $\beta$-driven neuroinflammation during autoimmune encephalomyelitis in mice. The Journal of experimental medicine 213 , 929-949 (2016).

30. Coll, R.C. et al. A small-molecule inhibitor of the NLRP3 inflammasome for the treatment of inflammatory diseases. Nature medicine 21 , 248-255 (2015).

31. Fleischman, A.G. et al. The CSF3R T618I mutation causes a lethal neutrophilic neoplasia in mice that is responsive to therapeutic JAK inhibition. Blood 122, 3628-3631 (2013).

32. Lin, X.L. et al. Heme oxygenase-1 directly binds STAT3 to control the generation of pathogenic Th17 cells during neutrophilic airway inflammation. Allergy 72, 1972-1987 (2017).

33. Paré, A. et al. IL-1 $\beta$ enables CNS access to CCR2(hi) monocytes and the generation of pathogenic cells through GM-CSF released by CNS endothelial cells. Proceedings of the National Academy of Sciences of the United States of America 115 , E1194-e1203 (2018).

34. Damasceno, L.E.A. et al. PKM2 promotes Th17 cell differentiation and autoimmune inflammation by fine-tuning STAT3 activation. The Journal of experimental medicine 217(2020).

35. Wu, B. et al. The TGF- $\beta$ superfamily cytokine Activin-A is induced during autoimmune neuroinflammation and drives pathogenic Th17 cell differentiation. Immunity 54, 308-323.e306 (2021).

36. Ciccia, F. et al. Type 3 innate lymphoid cells producing IL-17 and IL-22 are expanded in the gut, in the peripheral blood, synovial fluid and bone marrow of patients with ankylosing spondylitis. Annals of the rheumatic diseases $\mathbf{7 4}, 1739-1747$ (2015).

37. Hatfield, J.K. \& Brown, M.A. Group 3 innate lymphoid cells accumulate and exhibit disease-induced activation in the meninges in EAE. Cellular immunology 297, 69-79 (2015). 
38. Lv, J. et al. Airway epithelial TSLP production of TLR2 drives type 2 immunity in allergic airway inflammation. European journal of immunology 48, 1838-1850 (2018).

39. Martínez-López, M. et al. Microbiota Sensing by Mincle-Syk Axis in Dendritic Cells Regulates Interleukin-17 and -22 Production and Promotes Intestinal Barrier Integrity. Immunity 50 , 446-461.e449 (2019).

40. Haas, J., Korporal, M., Schwarz, A., Balint, B. \& Wildemann, B. The interleukin-7 receptor $\alpha$ chain contributes to altered homeostasis of regulatory T cells in multiple sclerosis. European journal of immunology 41, 845-853 (2011).

41. Eckhardt, J. et al. Thymic stromal lymphopoietin deficiency attenuates experimental autoimmune encephalomyelitis. Clinical and experimental immunology 181, 51-64 (2015).

42. Lee, H.C. et al. Hepatitis C virus promotes T-helper (Th)17 responses through thymic stromal lymphopoietin production by infected hepatocytes. Hepatology (Baltimore, Md.) 57 , 1314-1324 (2013).

43. Hirota, K. et al. Autoimmune Th17 Cells Induced Synovial Stromal and Innate Lymphoid Cell Secretion of the Cytokine GM-CSF to Initiate and Augment Autoimmune Arthritis. Immunity 48, 1220-1232.e1225 (2018).

44. Suto, H. et al. IL-25 enhances $\mathrm{T}(\mathrm{H}) 17$ cell-mediated contact dermatitis by promoting IL-1 $\beta$ production by dermal dendritic cells. The Journal of allergy and clinical immunology 142 , 1500-1509.e1510 (2018).

45. Wilson, S.R. et al. The epithelial cell-derived atopic dermatitis cytokine TSLP activates neurons to induce itch. Cell155 , 285-295 (2013).

46. Mishra, S.K. et al. Periostin Activation of Integrin Receptors on Sensory Neurons Induces Allergic Itch. Cell reports31, 107472 (2020).

47. Kitic, M. et al. Thymic stromal lymphopoietin is expressed in the intact central nervous system and upregulated in the myelin-degenerative central nervous system. Glia 62 , 1066-1074 (2014).

48. Hodecker, S.C. et al. Ruxolitinib treatment in a patient with neuromyelitis optica: A case report. Neurology(R) neuroimmunology \& neuroinflammation 4 , e328 (2017).

49. Wallace, D.J. et al. Baricitinib for systemic lupus erythematosus: a double-blind, randomised, placebocontrolled, phase 2 trial. Lancet (London, England) 392 , 222-231 (2018).

50. van der Heijde, D. et al. Tofacitinib in patients with ankylosing spondylitis: a phase II, 16-week, randomised, placebo-controlled, dose-ranging study. Annals of the rheumatic diseases $\mathbf{7 6}, 1340-1347$ (2017).

51. Schattling, B. et al. TRPM4 cation channel mediates axonal and neuronal degeneration in experimental autoimmune encephalomyelitis and multiple sclerosis. Nature medicine 18 , 1805-1811 (2012).

52. Fang, D. et al. Interleukin-6-mediated functional upregulation of TRPV1 receptors in dorsal root ganglion neurons through the activation of JAK/PI3K signaling pathway: roles in the development of bone cancer pain in a rat model. Pain 156, 1124-1144 (2015).

53. Malsch, P. et al. Deletion of interleukin-6 signal transducer gp130 in small sensory neurons attenuates mechanonociception and down-regulates TRPA1 expression. The Journal of neuroscience : the official journal of the Society for Neuroscience 34 , 9845-9856 (2014).

54. Torke, S. et al. Inhibition of Bruton's tyrosine kinase interferes with pathogenic B-cell development in inflammatory CNS demyelinating disease. Acta neuropathologica 140 , 535-548 (2020).

55. Montalban, X. et al. Placebo-Controlled Trial of an Oral BTK Inhibitor in Multiple Sclerosis. The New England journal of medicine 380 , 2406-2417 (2019).

\section{Abbreviations}


NLRP3: nucleotide-binding domain, leucine-rich repeat containing protein family, pyrin domain containing 3; TSLPR: thymic stromal lymphopoietin; JAK: Janus Kinase; CNS: Central Nervous System; EAE: experimentally atoimmune encephalomyelitis; STAT: Signal Transducer and Activator of Transcription; NMDAR: N-methyl-D-aspartate receptor; UVB: ultraviolet radiation b

\section{Conflict of interests}

All authors declare that they have no competing interests.

\section{Authors contributions}

Xueyuan Yu, Jiajia Lv, Jun Wu, and Yong Chen performed the experiments, data acquisition, analysis and interpretation and drafting the article. Fei Chen analyzed the data and critically revised the article. Li Wang designed the project and critically revised the article for the important intellectual content and final approval of the version to be published. All authors provided important review of the manuscript.

\section{Ethical statement}

This study was approved by the institutional research ethics committee of Nanjing Medical University affiliated Nanjing Brain Hospital.

Figure

\section{Fig 1}
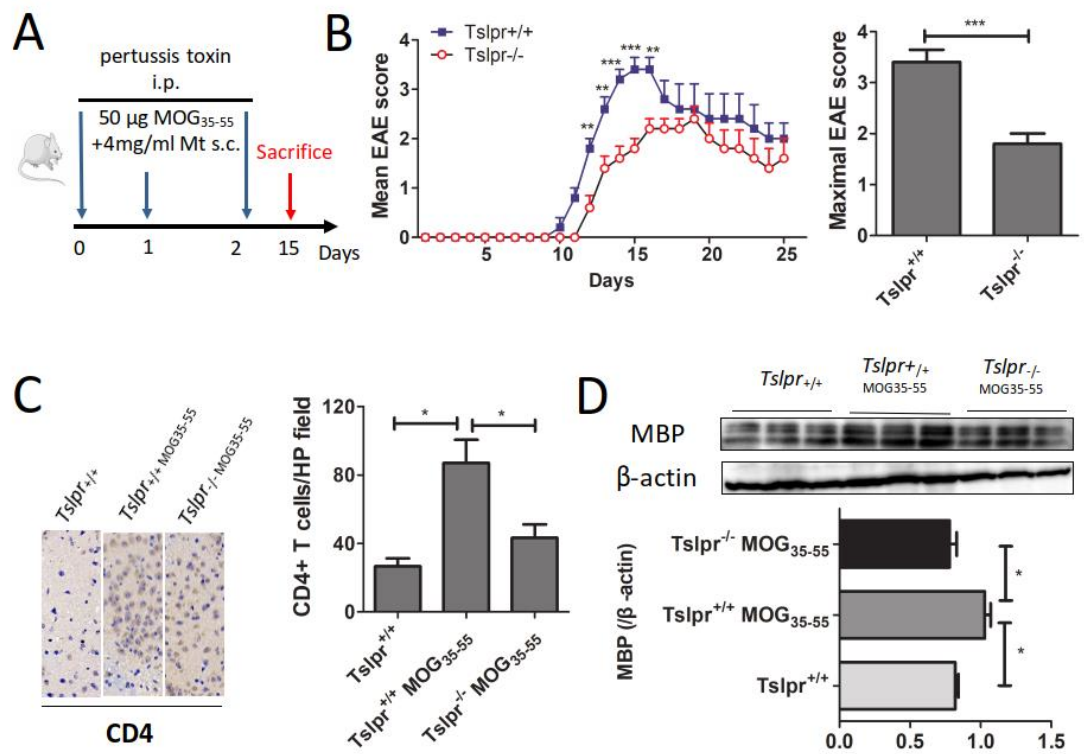

Figure 1. TSLPR deficiency alleviates neuroinflammation in EAE model. A, Schematic diagram of EAE mice model induction by subcutaneously injection of $\mathrm{MOG}_{35-55}$. B, EAE score in Tslpr ${ }^{-/-}$or Tslpr ${ }^{+/+}$mice ( $\mathrm{n}=5$ each group). C, CD4 staining of brain tissue of $T s l p r^{-/-}$and $T s l p r^{+/+}$miceafter EAE induction ( $\mathrm{n}=3$ each group). D, Expression of myelin basic protein (MBP) in mouse brain ( $\mathrm{n}=3$ each group). Data were presented as mean $\pm \mathrm{SEM}\left({ }^{*} p<0.05,{ }^{* *} p<0.01,{ }^{* * *} p<0.001\right)$ 
Fig 2
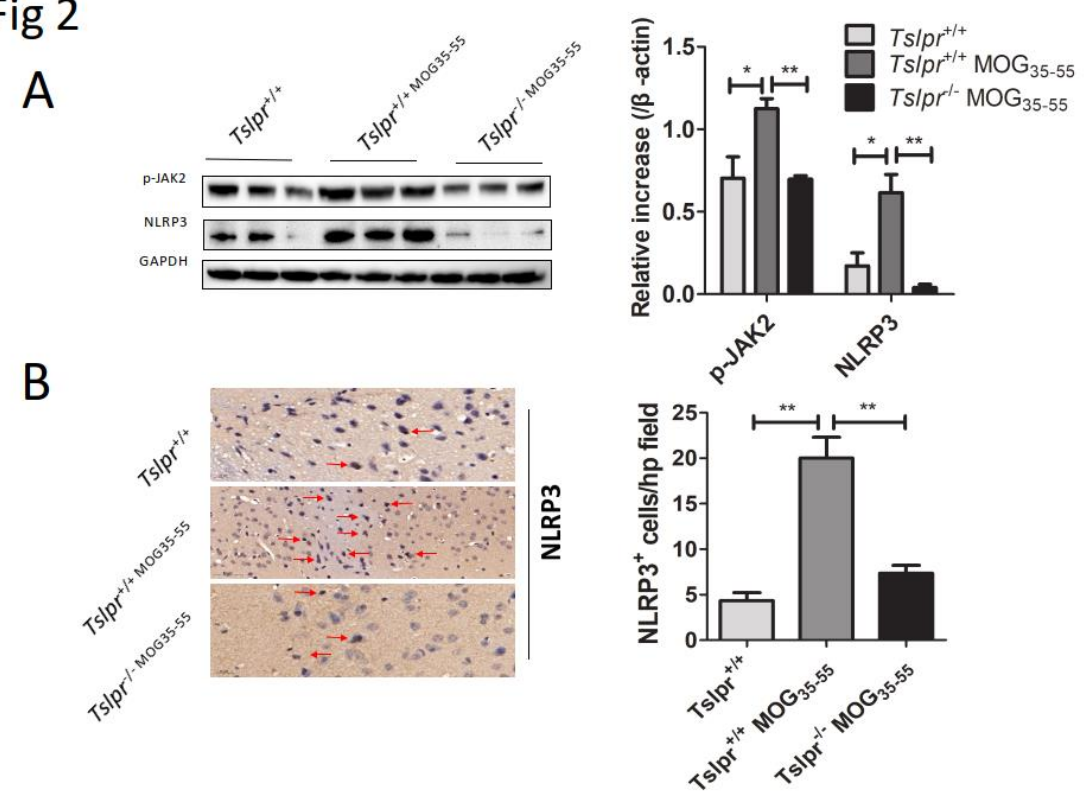

Figure 2. TSLPR signaling activates JAK2 and NLRP3 in EAE. A, Expression of NLRP3 and phosphorylation of JAK2 in brain tissues of $\mathrm{Tslpr}^{-/-}$and $\mathrm{Tslpr}^{+/+}$mice afetr EAE induction (n=3 each group). B, NLRP3 staining of brain tissue ( $\mathrm{n}=3$ each group). Red arrows indicate NLRP ${ }^{+}$cells. Data were presented as mean $\pm \operatorname{SEM}\left(\mathrm{n}=3,{ }^{*} p<0.05,{ }^{* *} p<0.05\right)$

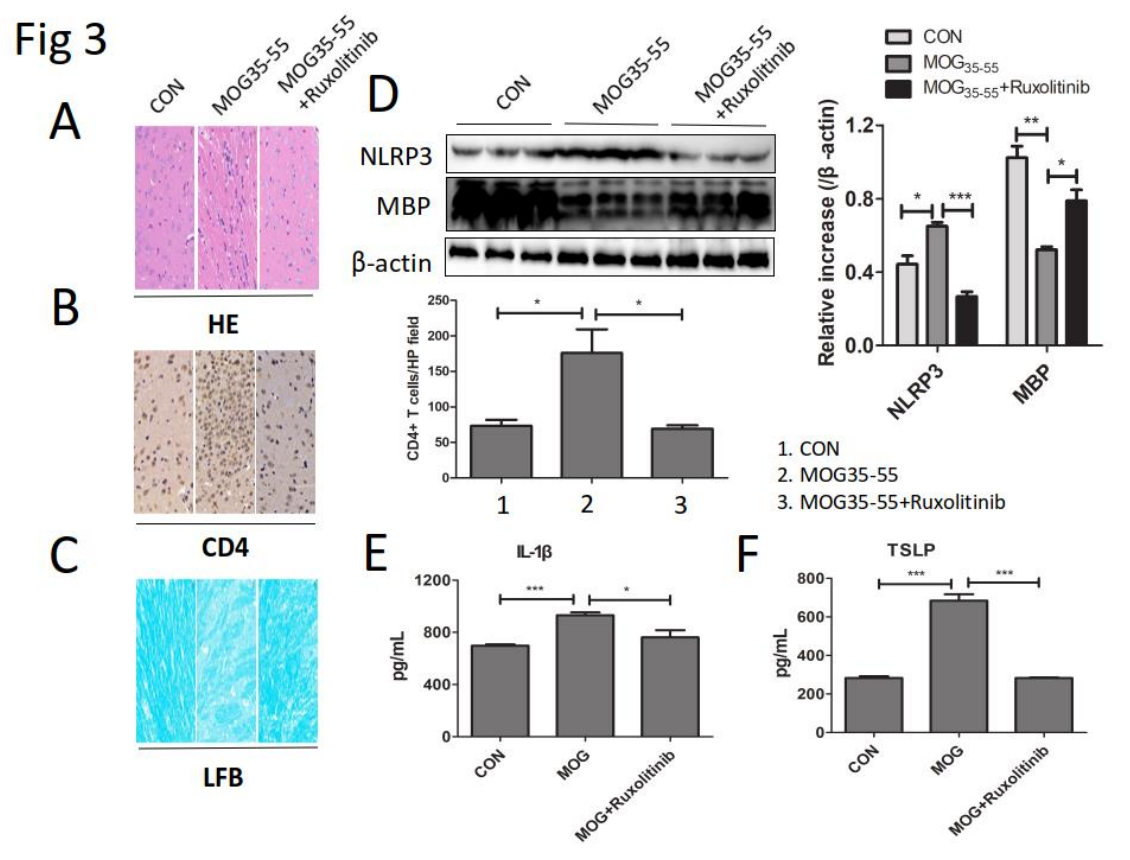

Figure 3. MOG $_{35-55}$-indcued neuroinflammation depends on JAK2 signaling. A, HE staining of mouse brain after induction of EAE in the absence or presence of ruxolitinibapplication. B, CD4 staining of 
mouse brain ( $\mathrm{n}=3$ each group). C, LFB staining of mouse brain. D, Expression of NLRP3 and MBP of mouse brain ( $n=3$ each group). E, Determination of IL- $1 \beta$ in mouse brain ( $n=4$ each group). F, Determination of TSLP in mouse brain ( $\mathrm{n}=4$ each group). Data were presented as mean \pm SEMin a high-power field (HPF) $\left({ }^{*} p<0.05,{ }^{* *} p<0.01,{ }^{* * *} p<0.001\right)$

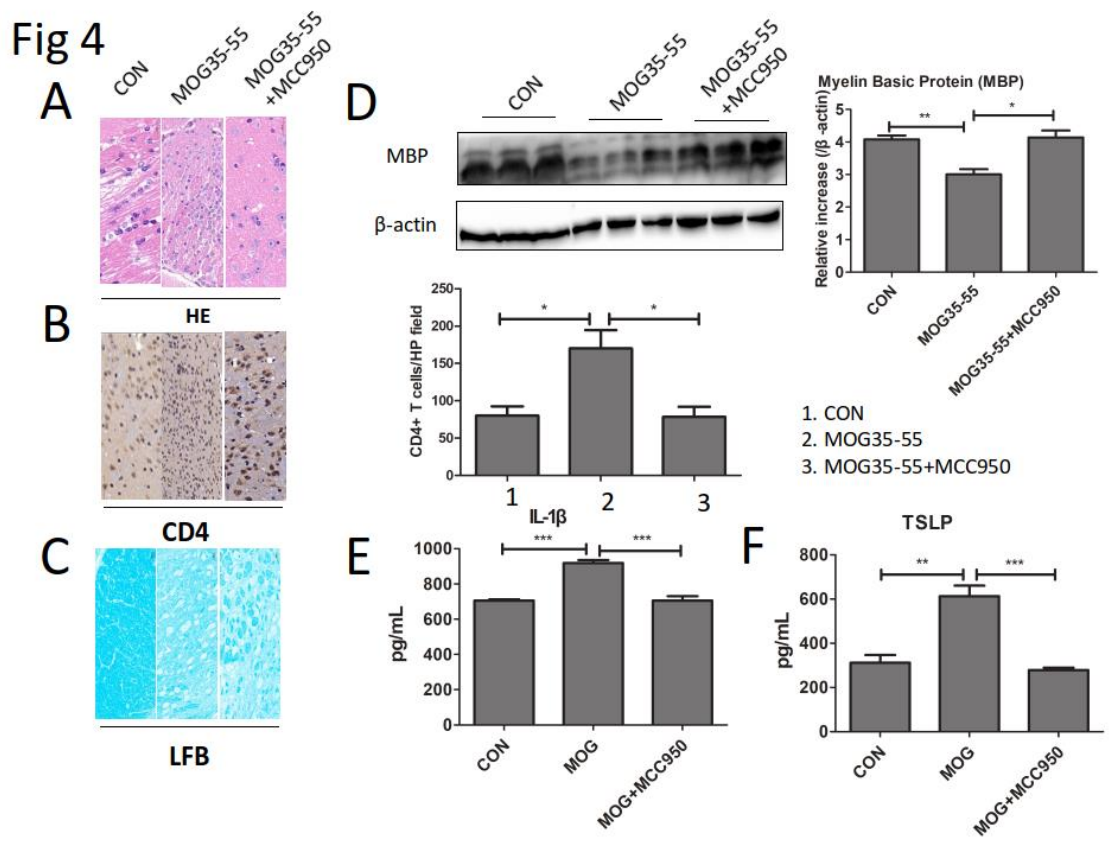

Figure 4. NLRP3 determins neuroinflammation of EAE. A, HE staining of mouse brain after induction of EAE in the absence or presence of MCC950 application. B, CD4 staining of mouse brain ( $\mathrm{n}=3$ each group). C, LFB staining of mouse brain. D, Expression of MBP of mouse brain ( $\mathrm{n}=3$ each group). E, Determination of IL- $1 \beta$ in mouse brain ( $\mathrm{n}=4$ each group). F, Determination of TSLP in mouse brain $(\mathrm{n}=4$ each group). Data were presented as mean \pm SEMin a high-power field (HPF) $\left({ }^{*} p<0.05,{ }^{* *} p<0.01,{ }^{* * *} p\right.$ $<0.001$ ) 
Fig 5

A

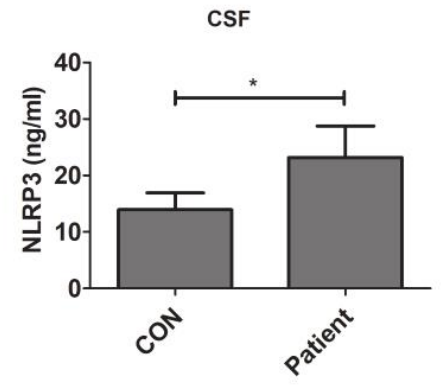

B

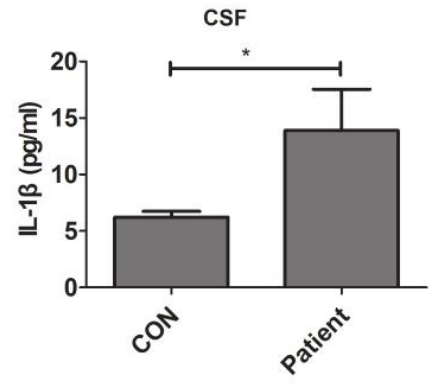

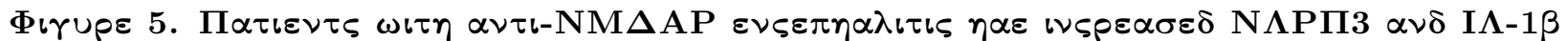
ఒ " $\boldsymbol{\Sigma} \boldsymbol{\Phi}$. A, Determination of NLRP3 inflammasome in CSF of patients with anti-NMDAR encephalitis $(\mathrm{n}=5)$ and control subjects $(\mathrm{n}=7)$ by ELISA. B, Determination of IL-1 $\beta$ inflammasome in CSF of patients with anti-NMDAR encephalitis $(\mathrm{n}=5)$ and control subjects $(\mathrm{n}=7)$ by ELISA. $\left({ }^{*} p<0.05\right)$

\begin{tabular}{|c|c|c|c|c|c|c|c|c|c|}
\hline & \multirow{2}{*}{$\begin{array}{l}\text { Age } \\
\text { (y) }\end{array}$} & \multirow{2}{*}{ Sex } & \multirow{2}{*}{ Disease } & \multicolumn{6}{|c|}{ CSF } \\
\hline & & & & $\begin{array}{c}\text { karyocyte } \\
\left(\times 10^{6} / \mathrm{L}\right)\end{array}$ & $\begin{array}{l}\text { Mononuc lear } \\
\text { cells (\%) }\end{array}$ & $\begin{array}{l}\text { Pro } \\
(\mathrm{g} / \mathrm{L})\end{array}$ & $\begin{array}{c}\text { Glu } \\
\text { (mmol/ } \\
\text { L) }\end{array}$ & $\begin{array}{l}\text { chloride } \\
(\text { mmol/L) }\end{array}$ & $\begin{array}{c}\text { Anti- } \\
\text { NMDAR } \\
\text { ant ibody }\end{array}$ \\
\hline Patient 1 & 17 & $\mathrm{~F}$ & $\mathrm{AE}$ & 51 & 99 & 0.35 & 3.2 & 121.7 & + \\
\hline Patient 2 & 19 & $\mathrm{~F}$ & $\mathrm{AE}$ & 0 & 0 & 0.4 & 3. 18 & 116. 5 & + \\
\hline Patient 3 & 25 & $\mathrm{~F}$ & $\mathrm{AE}$ & 0 & 0 & 0.41 & 4.4 & 120. 6 & + \\
\hline Patient 4 & 25 & $\mathrm{~F}$ & $\mathrm{AE}$ & 7 & 0 & 0.51 & 3. 18 & 119. 1 & + \\
\hline Patient 5 & 16 & M & $\mathrm{AE}$ & 6 & 0 & 0.42 & 3. 37 & 117.2 & + \\
\hline Control 1 & 65 & M & $\begin{array}{c}\text { Acute head } \\
\text { trauma }\end{array}$ & 0 & 0 & 0.77 & 2. 82 & 119. 7 & - \\
\hline Control 2 & 78 & M & Hydrocephalus & 1 & 0 & 0.56 & 3.14 & 119.3 & - \\
\hline Control 3 & 60 & $\mathrm{~F}$ & Hydrocephalus & 2 & 0 & 0.36 & 2. 63 & 117. 7 & - \\
\hline Control 4 & 51 & $\mathrm{~F}$ & Hydrocephalus & 0 & 0 & 0.19 & 3.51 & 125 & - \\
\hline Control 5 & 34 & M & Hydrocephalus & 3 & 0 & 0.35 & 2.51 & 118. 7 & - \\
\hline Control 6 & 82 & M & Hydrocephalus & 2 & 0 & 0.38 & 3.9 & 118.3 & - \\
\hline Control 7 & 75 & M & Hydrocephalus & 2 & 0 & 0.42 & 4.1 & 123 & - \\
\hline
\end{tabular}

F, female; M, male; $\mathrm{AE}$, autoimmune encephalitis; pro, protein; glu, glucose; CSF, cerebro-spinal fluid 